\title{
Note: Interference technique for minimally invasive, subnanometer, microsecond measurements of displacements
}

\author{
Irene Dujovne, ${ }^{1}$ Jacob Kerssemakers, ${ }^{1}$ G. Cappello, ${ }^{2}$ and Cees Dekker ${ }^{1}$ \\ ${ }^{1}$ Kavli Institute of NanoScience, Delft University of Technology, Lorentzweg 1, 2628 CJ Delft, \\ The Netherlands \\ ${ }^{2}$ Institut Curie, CNRS UMR 168, Paris 75248, France
}

(Received 29 September 2009; accepted 7 December 2009; published online 14 January 2010)

\begin{abstract}
We present a novel high-resolution technique for single-molecule experiments, viz., differential traveling wave tracking. This is an interference-based scattering technique where we use gold nanoparticles for high scattering intensities and incorporate differential measurements along one in-plane direction to subtract mechanical noise and drift of the system. In addition, out-of-plane distances are measured via scattered light intensity in a total internal reflectance illumination field. In plane, we demonstrate a rms noise level of only $0.10 \mathrm{~nm}$ at $10 \mathrm{kHz}$ and less than $0.5 \mathrm{~nm}$ at 600 kHz. () 2010 American Institute of Physics. [doi:10.1063/1.3280167]
\end{abstract}

Many biological processes involve a fast motion of, say, a protein, followed by a long dwell time. In order to study these processes, one needs to be able to probe different times scales, down to microseconds. Commonly used methods in single-molecule experiments have to either compromise on the temporal or the spatial resolution. ${ }^{1-5}$ Here, we present a technique with an exceptionally high spatial and temporal resolution that allows to probe at different timescales, which opens the door to the study of real-time dynamics of many biological processes. These include conformational changes of proteins, protein-DNA interactions, and rapid stepping of motor proteins, for example, kinesin on a microtubule, where the step takes less that $15 \mu$ s but dwell times are of the order of $10 \mathrm{~ms} .{ }^{3,6,7}$

Differential traveling wave tracking, the technique presented here, is a scattering technique based on the motion of a gold nanoparticle through a moving interference pattern. We perform differential measurements, where the relative displacements between two objects are determined, one moving and one fixed on the surface, which is used for reference. The one-dimensional displacements of the scattering objects are determined by measuring the phase shifts of the scattered signals with respect to the moving pattern ${ }^{8}$

$$
x=\phi \frac{d}{2 \pi},
$$

where $x$ is the displacement, $\phi$ is the phase shift, and $d$ is the periodicity given by a time-shifting interference pattern. We demonstrate the technique with the use of $\sim 80 \mathrm{~nm}$ gold nanoparticles that are much smaller than commonly used beads in other single molecule experiments. The use of $\mathrm{Au}$ nanoparticles with large scattering cross section as scatterer is important for high signal-to-noise ratios. Low powers are used for minimum invasiveness. The motion of the Au beads along $x$ and $z$ can be studied on their own, for example Brownian motion in biophysical systems, or beads can be attached to the protein under study by different ligands. In this paper, we describe the technique in detail and present an example of an application to the measurement of the tethered motion of short (224bp) DNA molecules.
Below, we demonstrate subnanometer spatial resolution and a long-term stability, tens of seconds, largely due to the implementation of a differential measurement. While this is easily implemented in imaging techniques, it has been elusive in scattering experiments with high temporal resolution. ${ }^{9}$ We accomplish it by spatially filtering the signal. Postprocessing of the data is performed using the fiducial (reference) mark to retrieve the carrier frequency. This signal not only has the information of the carrier frequency, $f_{c}$, but also information about the overall vibrations and drift of the system. The implementation of differential measurements and the use of gold as a scatterer allowed us to push the limits to subnanometer at timescales in excess of $400 \mathrm{kHz}$. In the planar component, we achieved $0.1 \mathrm{~nm}$ RMS noise at $10 \mathrm{KHz}$ with subnanometer drift over long (seconds) timescales.

Figure 1 displays the experimental configuration. A $532 \mathrm{~nm}$ laser with long coherence length is split into two components. These beams impinge onto two phase-locked acoustic-optic modulators (AOM) that provide a small frequency shift to each beam. One AOM modulates at a base frequency of $40 \mathrm{MHz}$ and the other one at a frequency of $40 \mathrm{MHz}+f_{c}$. The frequency difference, $f_{c}$, variable between $30 \mathrm{kHz}$ and $4 \mathrm{MHz}$, constitutes the carrier frequency, is limited by the resolution of the dual generator driving the AOMs. The beams are focused at the back focal plane of a 60 times, 1.45NA TIRF objective, in such a way that they have planar components that are equal in magnitude but in opposite direction when they emerge from the objective, thus creating a standing wave when the frequency shift between the beams is zero. The periodicity of this pattern is given by

$$
d=\frac{2 \pi}{k_{x}}=\frac{\lambda}{2 n_{g} \sin \theta},
$$

where $k_{x}$ is the wavevector in the plane at the glass/water interface, $\lambda$ is the laser wavelength, $n_{g}$ is the glass index of refraction, and $\theta$ is the angle of incidence with respect to the surface normal of the glass/water interface. ${ }^{8}$ The measured periodicity is $\sim 185 \mathrm{~nm}$. The beams emerge from the objective at an angle larger than the critical angle so that they 


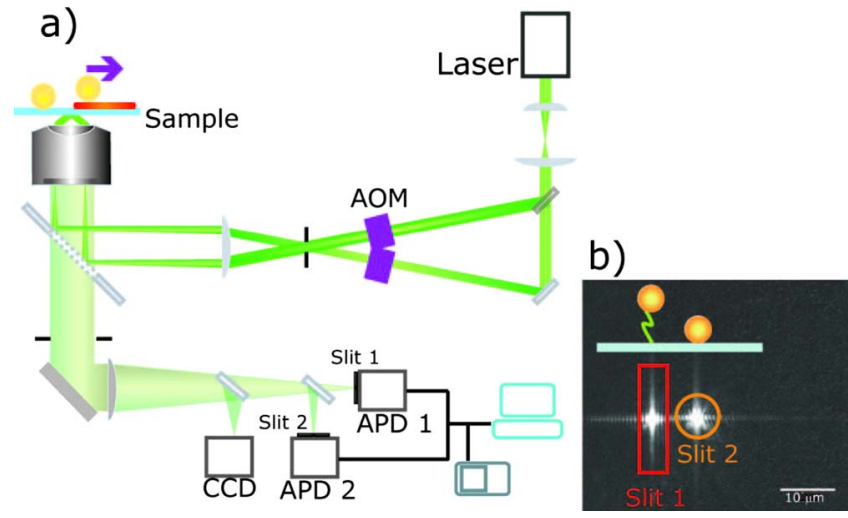

FIG. 1. (Color online) (a) Experimental configuration, (b) CCD image and representation of one experimental configuration with one tethered bead and one bead stuck to the glass surface. The rectangle and the circle represent the position of the two slits used to spatially filter the signal onto the APDs.

undergo total internal reflection (TIR) at the glass-water interface. Under TIR only a narrow strip of light ( $\$ 200 \mathrm{~nm}$ ) above the glass-water interface is illuminated by an exponentially decaying field, $I \propto \exp (-Z / \zeta)$, where $z$ is the distance away from the plane, $\zeta$ the penetration depth and $I$ the scattered light intensity. ${ }^{8}$ Therefore, the experiment determines the two-dimensional position of the scattering bead in $(x, z)$. The illuminated $(x, y)$ field of view is $\sim 20 \times 20 \mu \mathrm{m}^{2}$.

The scattering objects are gold nanoparticles ranging in diameter from 50 to $80 \mathrm{~nm}$ (Au50-Au80) either coated with goat anti-biotin antibodies or without coatings. The size and material of the nanoparticles used are such that their plasmon resonance is close to the laser wavelength used in this setup in order to obtain maximum scattering intensity. This, combined with the large scattering cross section of gold, allows us to use low powers $\left(\leqslant 0.025 \mathrm{~mW} / \mu \mathrm{m}^{2}\right.$ combined power intensity of the two beams in the illuminated area).

In order to produce our fiducial (reference) marks, $\sim 80 \mathrm{~nm}$ Au beads are deposited on the surface of a thin glass slide. Alternatively, a thin $(\sim 40 \mathrm{~nm})$ gold layer is evaporated on a clean thin glass slide, which is then locally stripped off, leaving small patches of gold on the surface. The sharp edges perpendicular to the direction of the traveling wave are a major source of scattering.

The scattered light is collected through the focusing objective and split into three detectors. First, a beam sampler is used to divert light to a CCD camera for the visualization of the experiment at long timescales $(10 \mathrm{~Hz})$. The remaining light is split by the use of a $50 / 50$ beam splitter into two point detectors that integrate the signal. These detectors are avalanche photodiodes (APDs) (10 MHz bandwidth). Each of these two detectors has a slit in front in order to be able to scan the image plane and choose the right acquisition position. This way, the reference signal is acquired by one detector and the signal of the moving object by the other. The minimal separation between the moving object and the reference object is given by the width of the slits and the magnification of the system. In our case, we can resolve items separated by a distance as close as $5 \mu \mathrm{m}$. The configuration could be extended by the use of additional APDs to track multiple beads, provided that these beads are optically separated in the projected image plane.
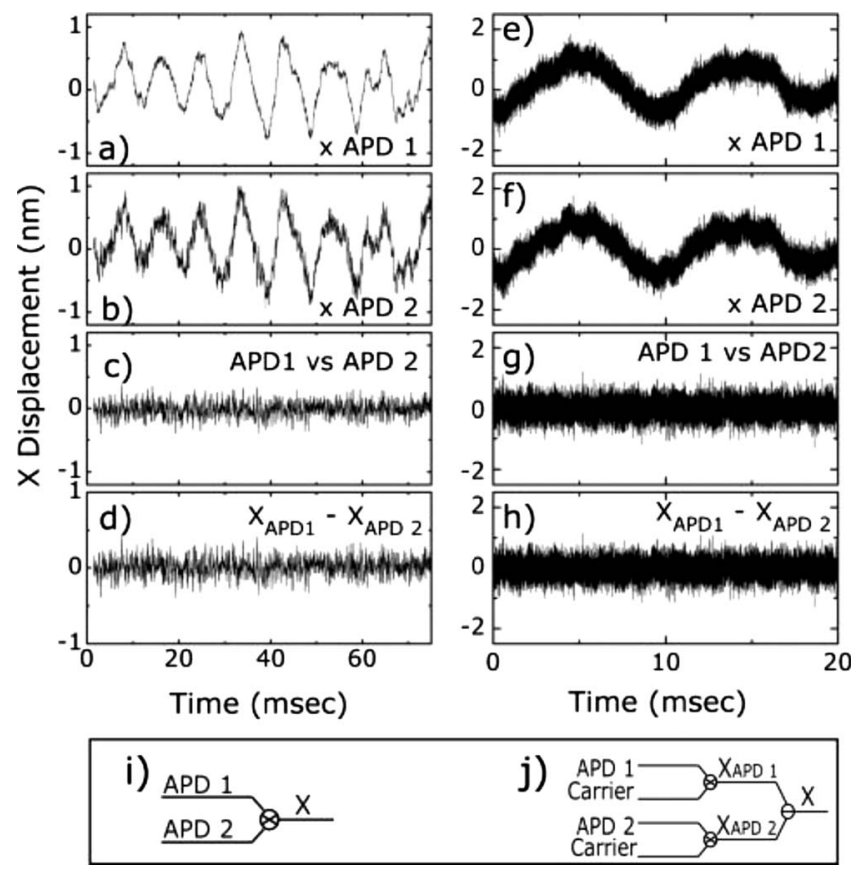

FIG. 2. Bead position as a function of time for a fixed bead on a glass surface. Both detectors, APD1 and APD2, image the displacement of the same object. (a)-(d) Data measured with a carrier frequency $f_{c}=40 \mathrm{kHz}$ and sampled at $750 \mathrm{kHz}$. (a) Position of the bead calculated using the signal from APD 1 as input vs $f_{c}$, (b) same, but now for APD2. As can be seen in the figures, the object oscillates with an amplitude of $\sim 2 \mathrm{~nm}$ peak-to-peak. These vibrations are due to building-related experimental conditions and are present permanently. (c) APD 1 locked-in on APD 2 [scheme (i)]. (d) Difference between the calculated displacements shown in (a) and (b) [scheme (j)]. As can be seen in (c) and (d), the vibrations disappear when calculating the displacement using the reference bead as reference signal and both methods yield a similar result (e)-(h), similar to (a)-(d) but measured with $f_{c}=1 \mathrm{MHz}$ and sampled at $50 \mathrm{MHz}$. After the calculation of the displacement as function of time, the signal is filtered down by the use of a Butterworth filter with a low pass frequency of $0.6 \mathrm{MHz}$. (i)-(j) Schematic of the analysis scheme for the data where $\otimes$ represent lock-in detection and $\ominus$ difference.

The simultaneous acquisition of the data from the photodiodes and dual generator is performed in three ways: either by a 4 channel oscilloscope (Lecroy Wavesurfer 6030, 8 bit, $50 \mathrm{MHz}$ bandwidth), a Linux based acquisition card (Spectrum GmbH, M2i.3122, 12 bit, $10 \mathrm{MHz}$ bandwidth), or by a data acquisition card (National Instruments, PCI-6120, 16 bit, $800 \mathrm{kHZ}$ bandwidth). The bandwidth is chosen in order to be able to acquire at least $20-25$ points per period of the carrier signal.

There are three collected signals: carrier, reference, and signal of interest. The carrier signal, $f_{c}$, is the difference between the two driving frequencies from the dual generator that are mixed and filtered by a custom-made mixer to provide the shifting interference signal electronically. The reference signal is the scattered light from the fiducial mark and contains the information of the vibrations and drift of the sample. Lastly, the signal of interest is the light scattered by the moving bead. These three signals are analyzed using a Matlab program which acts as a lock-in amplifier with either the carrier signal or the reference signal as the lock-in reference signal, see Fig. 2. The displacement $x$ is given by Eq. (1), while the displacement out of the plane is given by the intensity of the signal measured, viz., $z-z_{0}=-\zeta \ln (I)$. When both detectors are acquiring the signal of the same 

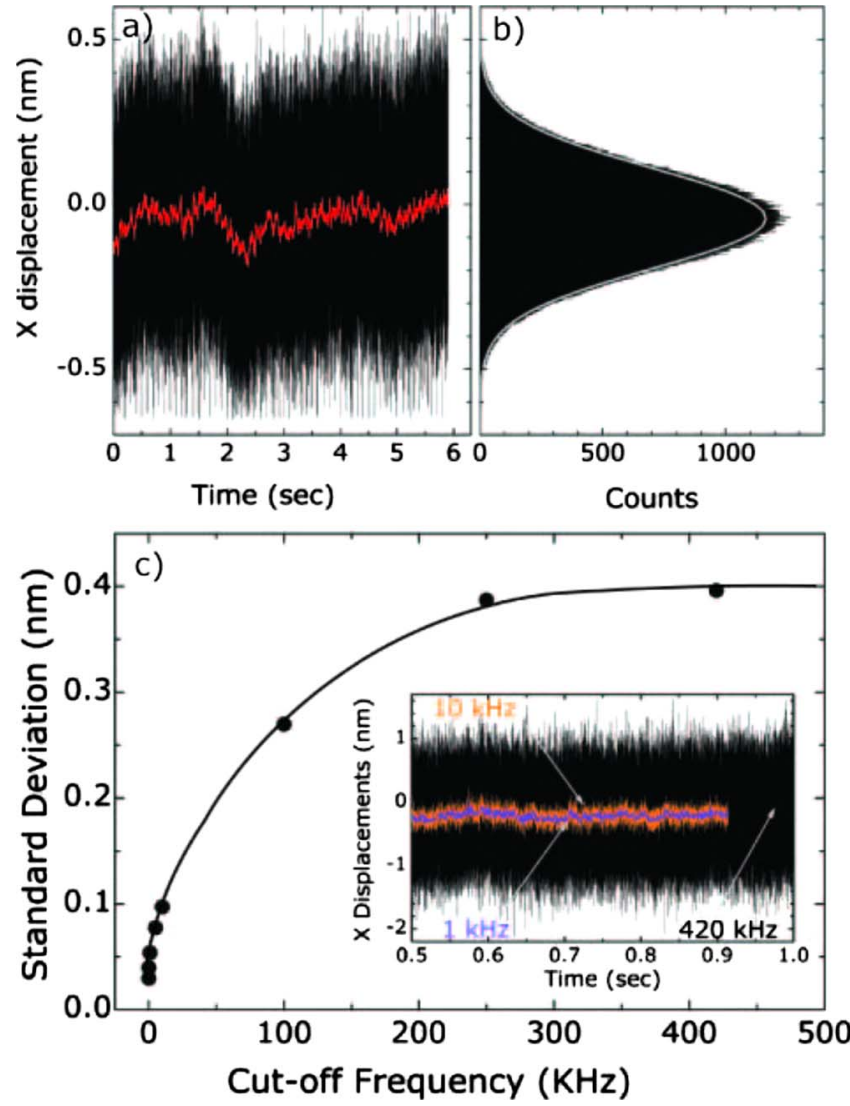

FIG. 3. (Color online) Long-term stability of the system. (a) Position as function of time of a fixed bead on a glass surface measured with both detectors on the same object. $f_{c}=40 \mathrm{kHz}$ and the acquisition was performed at $750 \mathrm{kHz}$. The red line is a moving average over $0.01 \mathrm{~s}$ of the data. (b) Histogram of the positions. The standard deviation of the data presented in (a) is $0.15 \mathrm{~nm}$ over the full six seconds. (c) Standard deviation of the position as function of time over $0.5 \mathrm{~s}$ for a fixed object with a $f_{c}=700 \mathrm{kHz}$ sampled at $10 \mathrm{MHz}$. The standard deviation is calculated after low-pass filtering the signal to the cut-off frequency (black $420 \mathrm{kHz}$, orange $10 \mathrm{kHz}$, purple $1 \mathrm{kHz}$. The inset displays the signal as function of time after low-pass filtering is applied.

object and we apply the lock-in technique on the signal from APD1 using the signal from APD 2 as reference, then the vibrations of the system are canceled out [Figs. 2(c) and $2(\mathrm{~g})]$. We are left with a very low residual RMS noise of $0.4 \mathrm{~nm}$ at $420 \mathrm{kHz}$. If the displacements are calculated independently using the carrier frequency as the lock-in signal, and then subtracted [Fig. 2(j)], we observe similar results, as shown in Figs. 2(d) and 2(h).

The long-term stability is displayed in Fig. 3(a) for $f_{c}=40 \mathrm{kHz}$, filtered with a low pass filter to $24 \mathrm{kHz}$. The stability is better than $0.3 \mathrm{~nm}$ over $6 \mathrm{~s}$ (taken as the full width of the distribution). The noise magnitude of the system is shown in Fig. 3(b). This was measured with a driving frequency, $f_{c}=700 \mathrm{kHz}$ and filtered down $420 \mathrm{kHz}$. In this figure, we present the standard deviation of the position as

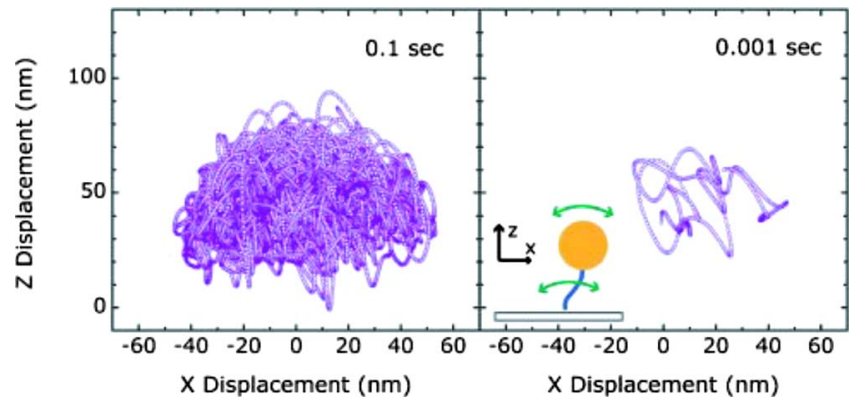

FIG. 4. (Color online) $Z$ displacement as function of $x$ displacement of a tethered gold particle. The tether length is 224 base pairs of dsDNA. (a) Total sampling time of $0.1 \mathrm{~s}$ (b) Total sampling time of $0.001 \mathrm{~s}$. The data presented here was measured at $40 \mathrm{kHz}$.

function of time over $0.5 \mathrm{~s}$ calculated after low-pass filtering the acquired data at different cut-off frequencies. As expected, the amplitude of the RMS noise diminishes with the filtering, starting at $0.4 \mathrm{~nm}$ at the higher frequencies down to values of $0.10 \mathrm{~nm}$ at $10 \mathrm{KHz}$ and $0.05 \mathrm{~nm}$ at $1 \mathrm{kHz}$.

In order to exemplify the capabilities of the system, we performed tethered-particle-motion studies of short strands of double stranded DNA (dsDNA) (Dujovne et al. in preparation). Figure 4 displays $z-x$ displacements for a dsDNA of 224 base pairs long. Figure 4(a) displays $z-x$ position sampled over a period of $0.1 \mathrm{~s}$, while Fig. 4(b) displays only a $0.001 \mathrm{~s}$ interval. As can be seen from this figure, the position of the nanoparticle can be determined with high precision even at very short timescales.

The low displacement noise and high bandwidth of our technique opens the door to the study of real time dynamics of, for example, DNA-protein interactions where the change in the length of dsDNA due to the interactions with a protein could be followed in real time down to the microsecond regime. The technique presented here for tracking a single moving particle at high resolution can be expanded to track multiple, optically separated particles by employing as many more separate detector devices, creating a many-particle tracking system with high spatial and temporal resolution.

${ }^{1}$ R. E. Thompson, D. R. Larson, and W. W. Webb, Biophys. J. 82, 2775 (2002).

${ }^{2}$ A. Yildiz, J. N. Forkey, S. A. McKinney, T. Ha, Y. E. Goldman, and P. R. Selvin, Science 300, 2061 (2003).

${ }^{3}$ S. M. Block, C. L. Asbury, J. W. Shaevitz, and M. J. Lang, Proc. Natl. Acad. Sci. U.S.A. 100, 2351 (2003).

${ }^{4}$ L. Nugent-Glandorf and T. T. Perkins, Opt. Lett. 29, 2611 (2004).

${ }^{5}$ E. Toprak and P. R. Selvin, Annu. Rev. Biophys. Biomol. Struct. 36, 349 (2007).

${ }^{6}$ N. J. Carter and R. A. Cross, Nature (London) 435, 308 (2005).

${ }^{7}$ E. A. Abbondanzieri, W. J. Greenleaf, J. W. Shaevitz, R. Landick, and S. M. Block, Nature (London) 438, 460 (2005).

${ }^{8}$ L. Busoni, A. Dornier, J.-L. Viovy, J. Prost, and G. Cappello, J. Appl. Phys. 98, 064302 (2005).

${ }^{9}$ G. Cappello, P. Pierobon, C. Symonds, L. Busoni, J. Christof, M. Gebhardt, M. Rief, and J. Prost, Proc. Natl. Acad. Sci. U.S.A. 104, 15328 (2007). 